\title{
Biliary mucinous cystic neoplasm with invasive carcinoma and recurrent cholangitis due to hepatolithiasis
}

\begin{abstract}
Biliary mucinous cystic neoplasms are very rare tumors. They are thought to be occurred due to malignant transformation of biliary cystadenoma. Biliary mucinous cystic neoplasm is commonly seen in women and more prevalent in fifth decade of life. Preoperative differential diagnosis of hepatic atypical cystic lesion is difficult. Biliary cystadenocarcinomas are usually multiloculated cystic lesions. Typical imaging characteristics are the existence of thick wall, thick and irregular internal septations, intramural nodules, papillary projections and contrast enhancement. However, they can appear with nonspecific imaging findings. Biliary cystadenoma and cystadenocarcinoma can mimic the imaging features of other cystic liver lesions. Therefore, the imaging findings of this rare case with the biliary mucinous cystadenocarcinoma associated with recurrent cholangitis and hepatolithiasis were presented.
\end{abstract}

Keywords: biliary mucinous cystic neoplasm, cholangitis, hepatolithiasis, magnetic resonance imaging
Volume 12 Issue 3 - 202I

\author{
Fatma Kulali \\ Associate Professor of Radiology Department, University of \\ Health Sciences Umraniye Training and Research Hospital, \\ Turkey
}

Correspondence: Fatma Kulali, Adem Yavuz Street. No: I, University of Health Sciences, Umraniye Training and Research Hospital, Department of Radiology, Umraniye, 34764, Istanbul, Turkey, Tel +90 (216) 632 18I8*487I, Fax +90 (216) 6327। 24 , Email ftkulali@gmail.com

Received: June 03,202I | Published: June 15, 2021

\section{Introduction}

Biliary mucinous cystic neoplasm (BCMN) is slow growing extremely rare tumor. ${ }^{1,2}$ They originate from the bile duct and include epithelial cells producing mucin. ${ }^{1-3}$ The pathophysiology and etiology are uncertain but the malignant transformation of biliary cystadenomas or de novo development can be a reason. ${ }^{1}$ BCMNs are commonly seen in women and mostly presented in fifth decade of life. ${ }^{2}$ According to World Health Organization (WHO) 2010 histological classification, they have been categorized into noninvasive and invasive types. ${ }^{3}$ Non-invasive BCMNs are subdivided according to the degree of epithelial dysplasia into three grades: low, intermediate and high. Invasive type is defined as BCMN with associated invasive carcinoma. The appropriate treatment modality is surgery for non-invasive and invasive types of BCMNs because of the risk of malignant transformation. Surgical excision is the most appropriate treatment in patients with $\mathrm{BCMN} .{ }^{3}$ The imaging findings of BCMNs can mimic other cystic hepatic lesions. Therefore, early diagnosis is difficult. ${ }^{1,3}$ The imaging modalities like ultrasonography (US), computed tomography (CT) or magnetic resonance imaging (MRI) can be performed for diagnosis of BCMNs. Some imaging findings such as thick wall, thick and irregular septa, the presence of intramural nodule or papillary projections and the contrast enhancement generally exhibit high risk of malignancy. ${ }^{3-6}$ Sometimes, the existence of non-specific imaging features makes it difficult to differentiate non-invasive type from invasive type of BCMN. For this reason, we aimed to present $\mathrm{CT}$ and MRI findings of an extreme case of biliary mucinous cystic neoplasm with associated invasive carcinoma combined with recurrent cholangitis due to hepatolithiasis.

\section{Case report}

A 75-year-old female patient referred to our institution with fever and abdominal pain. She had history of cholecystectomy and recurrent cholangitis due to biliary stones for three years. There was only right upper quadrant abdominal pain in her physical examination. Laboratory findings revealed high white blood count, C-reactive protein, aspartate transaminase, alanine transaminase, gamma-glutamyl transpeptidase, alkaline phosphatase and lactate dehydrogenase levels with normal bilirubin levels. Except for elevated CEA levels, tumor markers such as Ca-125, Ca 15-3, Ca 19-9 were normal. Non-contrast and contrast enhanced abdominal CT was performed. Dilatation of intrahepatic and extrahepatic bile ducts were detected on CT. Multiple stones inside bilateral intrahepatic bile ducts and common bile duct were seen on non-contrast axial CT images (Figure 1). There was a clustered, dilated left intrahepatic bile ducts forming a mass like appearance on left lobe of liver. Stones were also seen in this mass like clustered dilated intrahepatic bile ducts. Dilated intrahepatic bile ducts had thick wall and contrast enhancement. Abscess formations were found near mass like clustered dilated bile ducts. To characterize mass like appearance, contrast enhanced MRI was performed. Dilatation of intrahepatic and extrahepatic bile ducts, multiple stones inside them and clustered mass like appearance of bile ducts on left lobe were visualized better (Figure 2, Figure 3). The clustered dilated bile ducts on left lobe had no solid component. Thick walls of bile ducts had contrast enhancement. When we compared these imaging findings with previous CT and MRI examinations three years ago, mass like appearance was also seen on previous imaging. There was no significant progression without abscess formations and number of stones inside the biliary system. After antibiotic treatment, regression of abscess formations was detected on follow-up CT. Although no progression on mass like appearance of dilated intrahepatic bile ducts on left lobe of liver compared to previous examinations three years ago, tru-cut biopsy was performed to differentiate chronic sequel of recurrent cholangitis from tumor. Histopathological result was BCMN with invasive carcinoma according to WHO 2010 histopathological classification. 


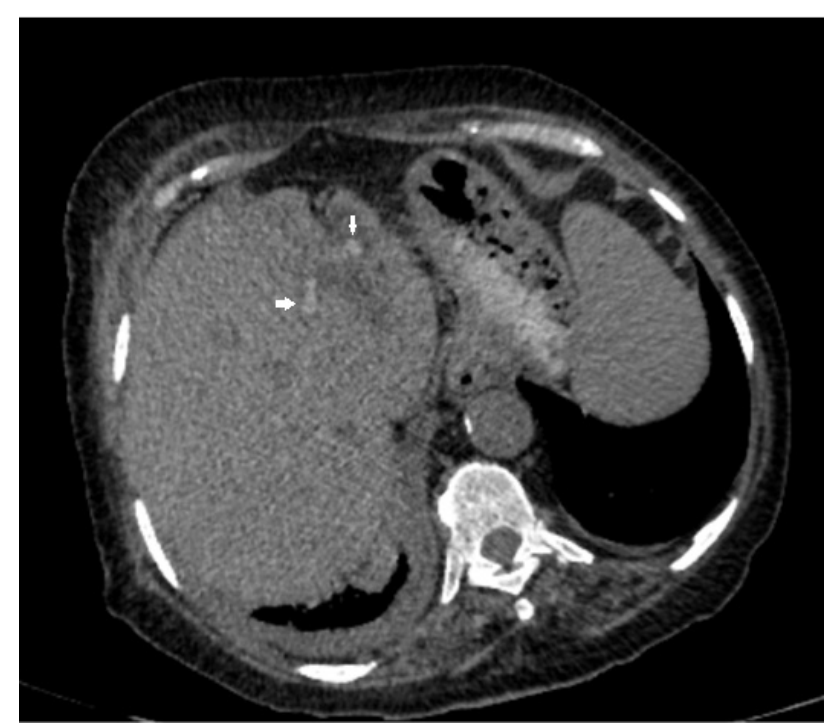

Figure I Hyperdense hepatolithiasis (arrows) and dilated intrahepatic bile ducts on axial non-contrast CT image.

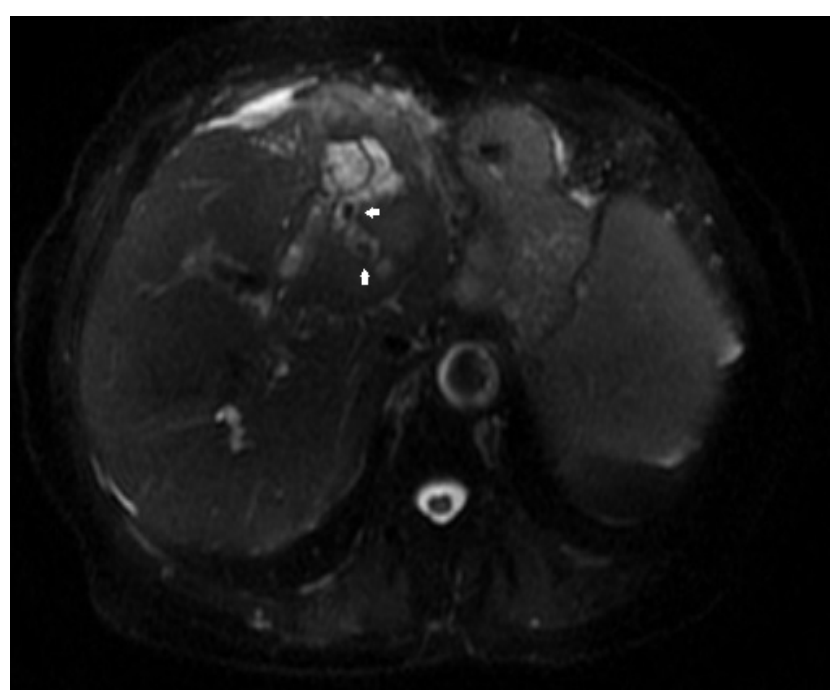

Figure 2 Hypointense hepatolithiasis (arrow) inside the dilated intrahepatic bile ducts on axial fat-saturated T2-weighted sequence.

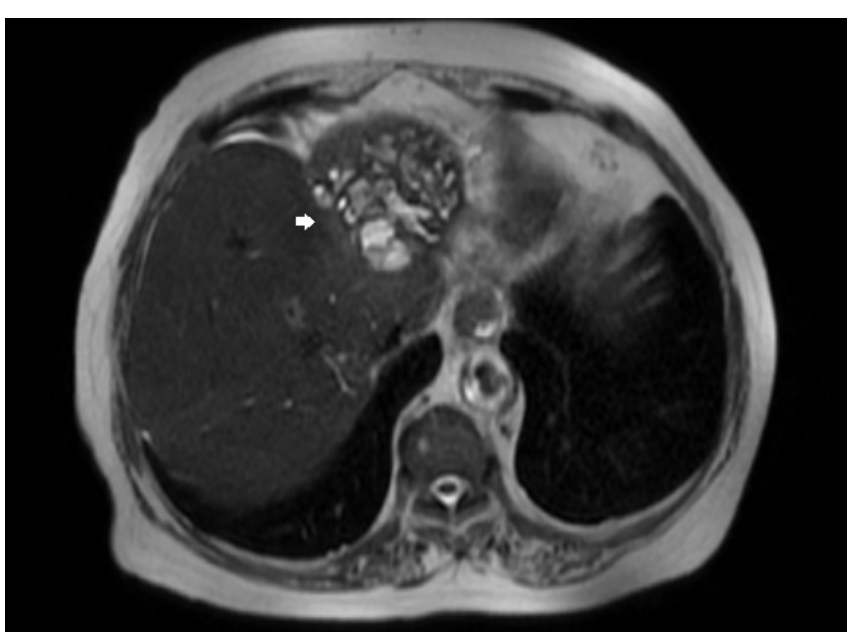

Figure 3 Clustered mass like appearance of bile ducts (arrow) on axial T2weighted sequence.

\section{Discussion}

Hepatolithiasis is more prevalent in Asian countries with 2\%-25\% incidence rates. ${ }^{7}$ Hepatolithiasis, cholestasis, recurrent cholangitis and biliary cysts can lead to malignancy.,5 Older age, biliary stricture, liver atrophy, high CA 19-9 level, left sided hepatolithiasis, stone recurrence and choledocho-enterostomy increase the risk of malignancy in patients with hepatolithiasis. In patients with hepatolithiasis, the long-term duration of symptom is an important risk factor for malignancy. ${ }^{10-15}$

BCMNs with invasive carcinoma are extremely rare with incidence of approximately $1 / 10.000 .000 .^{2}$ The pre-operative imaging modalities are important for early diagnosis and planning treatment. US, CT or MRI can be performed for diagnosis. Thick wall or septa, intramural nodule or papillary projections irregular contour and contrast enhancement are malignant imaging features of BCMNs. ${ }^{3,4}$ Calcification can also be seen. The diagnostic accuracy of BCMNs on $\mathrm{CT}$ or MRI with the benefit of contrast enhancement is higher compared to US. However, benign and malignant BCMNs can cause the same imaging findings. In the absence of mural nodule, papillary projection or contrast enhancement, the differential diagnosis can be difficult. ${ }^{1,3}$ However, some hepatic lesions such as hepatic abscess, complicated cysts or infected hydatid cysts can mimic BCMNs. Hepatic abscess and infected hydatid cysts may have thick wall with contrast enhancement. Complicated cysts may also show contrast enhancement of wall and septa. Enhanced solid component is not observed inside hepatic abscess or complicated cysts. Hydatid cysts may have solid content without contrast enhancement. The history and clinical findings of patients associated with imaging findings are necessary to make accurate diagnosis. But, in the case of recurrent cholangitis, it is hard to differentiate pseudo-lesions due to benign biliary strictures and hepatic abscess formations from cystic tumors. In patients with suspicious findings, tru-cut biopsy or surgery should be performed.

In patients with recurrent cholangitis, it is difficult to distinguish benign lesions from malignant ones due to distortion of normal anatomy and chronic fibrotic changes. Awareness of the possibility of malignant $\mathrm{BCMN}$ is important in patients with recurrent cholangitis, even though no progression of lesion compared to previous modalities and similar appearance with focal dilated biliary ducts and fibrotic changes may be observed on MRI.

\section{Acknowledgments}

This research did not receive any specific grant from funding agencies in the public, commercial, or not-for-profit sectors.

\section{Declarations of interest}

None.

\section{References}

1. Yang-Hong Dai, Yee Hui Yeo, Yao-Feng Li, et al. Hepatobiliary cystadenocarcinoma without mesenchymal stroma in a female patient: a case report. BMC Gastroenterol. 2014;14:109.

2. Kim JY, Kim SH, Eun HW, et al. Differentiation between biliary cystic neoplasms and simple cysts of the liver: accuracy of CT. AJR Am J Roentgenol. 2010;195(5):1142-1148.

3. Thomas KT, Welch D, Trueblood A, et al. Effective treatment of biliary cystadenoma. Ann Surg. 2005;241(5):769-773.

4. Simo KA, Mckillop IH, Ahrens WA, et al. Invasive biliary mucinous cystic neoplasm: a review. HPB (Oxford). 2012;14(11):725-740. 
5. Yamashita S, Tanaka N, Takahashi $M$, et al. Clinicopathological subclassification of biliary cystic tumors: Report of 4 cases with a review of the literature. Intractable Rare Dis Res. 2013;2(2):63-68.

6. Erdogan D, Busch OR, Rauws EA, et al. Obstructive jaundice due to hepatobiliary cystadenoma or cystadenocarcinoma. World J Gastroenterol. 2006;12(35):5735-5738.

7. Safari MT, Shahrokh S, Miri MB, et al. Biliary mucinous cystic neoplasm: a case report and review of the literature. Gastroenterol Hepatol Bed Bench. 2016;9(Suppl 1):S88-S92.

8. Wilkinson N. Hepatobiliary cystadenocarcinoma. Case Reports Hepatol. 2012;2012:298957.

9. Kim HJ, Kim JS, Joo MK, et al. Hepatolithiasis and intrahepatic cholangiocarcinoma: A review. World $J$ Gastroenterol. 2015;21(48):13418-13431.

10. Kim YT, Byun JS, Kim J, et al. Factors predicting concurrent cholangiocarcinomas associated with hepatolithiasis. Hepatogastroenterology. 2003;50(49):8-12.
11. Liu ZY, Zhou YM, Shi LH, et al. Risk factors of intrahepatic cholangiocarcinoma in patients with hepatolithiasis: a case-control study. Hepatobiliary Pancreat Dis Int. 2011;10(6):626-631.

12. Tsui WM, Chan YK, Wong CT, et al. Hepatolithiasis and the syndrome of recurrent pyogenic cholangitis: clinical, radiologic, and pathologic features. Semin Liver Dis. 2011;31(1):33-48.

13. Suzuki Y, Mori T, Abe N, et al. Predictive factors for cholangiocarcinoma associated with hepatolithiasis determined on the basis of Japanese Multicenter study. Hepatol Res. 2012;42(2):166-170.

14. Bettschart V, Clayton RA, Parks RW, et al. Cholangiocarcinoma arising after biliary-enteric drainage procedures for benign disease. Gut. 2002;51(1):128-129.

15. Zhang XJ, Jiang Y, Wang X, et al. Comparatively lower postoperative hepatolithiasis risk with hepaticocholedochostomy versus hepaticojejunostomy. Hepatobiliary Pancreat Dis Int. 2010;9(1):38-43. 